\title{
Cannabinoid Hyperemesis Syndrome Presenting With Recurrent Acute Renal Failure
}

\author{
Oladapo A Abodunde ${ }^{\mathrm{a}, \mathrm{c}}$, Joseph Nakda ${ }^{\mathrm{a}}$, Nneka Nweke ${ }^{\mathrm{a}}$, Raghava Levaka Veera ${ }^{\mathrm{b}}$
}

\begin{abstract}
Cannabinoid hyperemesis syndrome is a recently recognized clinical syndrome characterized by chronic cannabis use, profound vomiting and compulsive bathing among other features. Despite the degree of vomiting associated with this disorder, renal impairment and electrolyte disturbances are not commonly reported findings. We report the case of a 36-year-old male with a history of chronic daily cannabis use, who presented to our hospital with acute renal impairment five times in 4 years, with nearly identical clinical picture in each presentation. His symptom complex in each admission consisted of severe nausea and vomiting, compulsive hot showers, and resolution of symptoms within days of abstaining from cannabis. His clinical course included significantly abnormal basic metabolic panels at the time of each admission, with return to normal baseline values 96 hours following hydration in every case. The other consistent findings on admission were hypochloremic, hyponatremic dehydration with mixed metabolic alkalosis and high anion gap metabolic acidosis. He also had proteinuria and microscopic hematuria.This case highlights a serious and potentially life-threatening complication of the disorder. The consistency of the findings suggests a pattern that is associated with this disorder. It is unclear whether the course of renal involvement is benign in the long term.
\end{abstract}

Keywords: Cannabinoid; Cyclical vomiting; Renal failure

\section{Introduction}

Severe nausea and vomiting have long been recognized as

\footnotetext{
Manuscript accepted for publication October 25, 2012

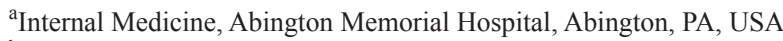

${ }^{\mathrm{b}}$ Medicine, Abington Memorial Hospital, Abington, PA, USA

${ }^{\mathrm{c}}$ Corresponding author: Oladapo A Abodunde, Internal Medicine, Abington Memorial Hospital, Abington, PA, 19001, USA.

Email: abodundedapo1@yahoo.com
}

doi: http://dx.doi.org/10.4021/jmc939w the predominant symptoms in several clinical syndromes, including cyclical vomiting syndrome, gastroparesis and hyperemesis gravidarum. A newly recognized syndrome in which these symptoms are prominent is the cannabinoid hyperemesis syndrome (CHS), characterized by chronic cannabis use, profound vomiting and compulsive bathing among other features. Although renal involvement and electrolyte disturbances would be expected given the risk of dehydration, acute renal failure has not been reported as a major feature of this syndrome.

\section{Case Report}

Cannabinoid hyperemesis syndrome (CHS) is a recently recognized clinical syndrome characterized by chronic cannabis use, profound vomiting and compulsive bathing among other features. Renal impairment and electrolyte disturbances have not been reported as prominent features.

A 36-year-old male presented to our hospital with acute renal impairment five times in 4 years. He first presented at the age of 31 years with a one-week history of severe nausea and vomiting. He had no significant past medical history. Creatinine on admission was $4.1 \mathrm{mg} / \mathrm{dl}$ and blood urea nitrogen (BUN) $71 \mathrm{mg} / \mathrm{dl}$ (Table 1). Extensive evaluation was performed, including anti-nuclear antibodies (ANA), hepatitis B surface antigen (HBsAg), anti-hepatitis C virus antibody (anti-HCV), serum protein electrophoresis (SPEP) and serum immunoelectrophoresis (SIEP). Anti-streptolysin $\mathrm{O}$ (ASO) titer, urine drug screen (including amphetamine, barbiturate, benzodiazepine, opioids and cocaine), anti-proteinase-3 (PR-3) antibody, anti-myeloperoxidase (MPO) antibody, complement components $\mathrm{C} 3, \mathrm{C} 4$ and $\mathrm{CH} 50$ were also assayed and all tests yielded normal results. Ultrasound of the kidneys revealed no hydronephrosis. Urinalysis showed trace protein, ketones and blood. Within two days, his serum creatinine had returned to normal and he was discharged. The only intervention was hydration with intravenous fluids. He was seen in the emergency department two years after his initial hospitalization for unrelated reasons, and serum creatinine checked at that time was normal.

Four years after the first admission, he presented again 


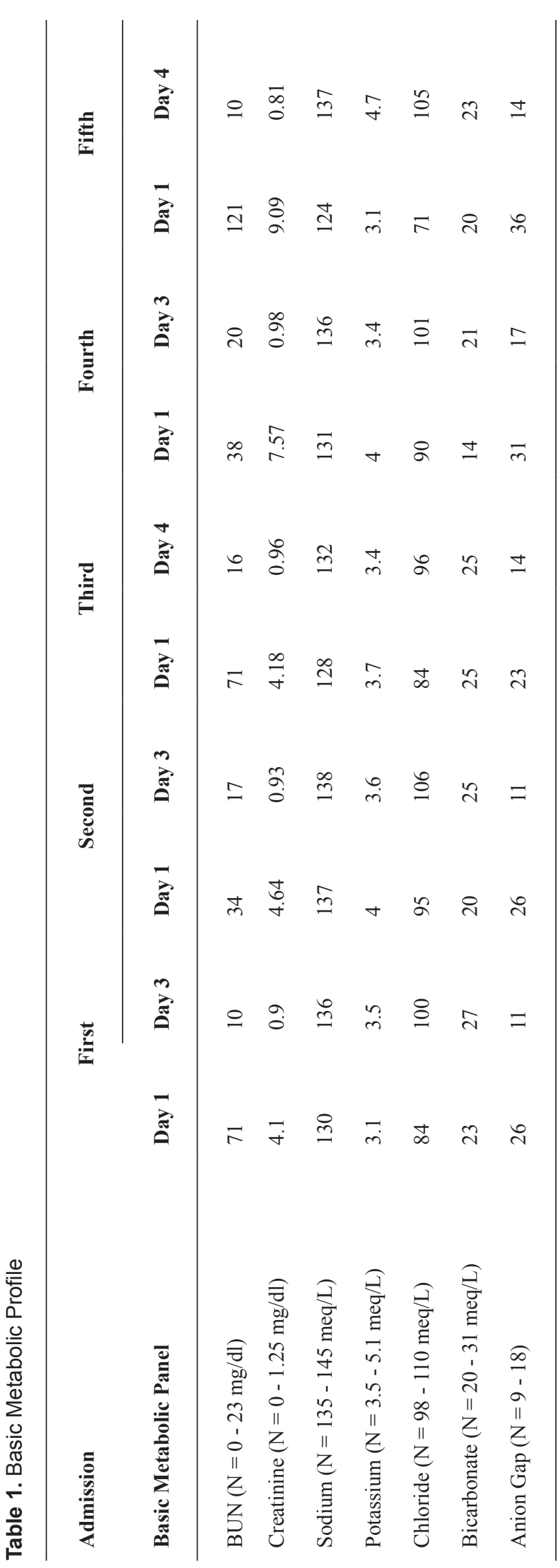

with severe nausea and vomiting. The only addition to his medical history was a diagnosis of psoriatic arthritis within the past 2 years, and he had been receiving infliximab. He was also taking meloxicam for pain. Creatinine was 4.64 $\mathrm{mg} / \mathrm{dl}$ and BUN $34 \mathrm{mg} / \mathrm{dl}$ (Table 1). He also stated that he used marijuana regularly, and in subsequent admissions, admitted to using marijuana regularly since high school. Following hydration with intravenous fluids, his BUN and creatinine returned to reference limits within two days and he was discharged with advice to avoid non-steroidal antiinflammatory drugs. He however returned five days later with severe nausea and vomiting. He denied taking any alcohol but had smoked marijuana daily since leaving hospital. BUN was $71 \mathrm{mg} / \mathrm{dl}$, creatinine $4.18 \mathrm{mg} / \mathrm{dl}$ (Table 1). With intravenous hydration, creatinine was $1.99 \mathrm{mg} / \mathrm{dl}$ on day 2 and by day 4, it was normal. Esophagogastroduodenoscopy (EGD) was performed for evaluation of nausea and vomiting, and the only abnormal findings were mild esophagitis and gastritis.

Three months after his third admission, he came to the emergency department for the fourth time complaining of four days of malaise and sore throat and two days of persistent nausea and vomiting. He also complained of abdominal pain but computerized tomography of the abdomen revealed no findings to explain his symptoms. Serum lipase and amylase were within reference limits. Creatinine on admission was $7.57 \mathrm{mg} / \mathrm{dl}$ and BUN was $38 \mathrm{mg} / \mathrm{dl}$ (Table 1). Two days later, BUN and creatinine were normal following intravenous hydration alone. During this admission, he had detailed re-evaluation including ASO titer, ANA, HBsAg, anti-HCV, SIEP, serum immunoglobulin assays, PR-3 antibody, MPO antibody, C3, C4, CH50, cryoglobulins and anti-glomerular basement membrane (GBM) antibody. All were within normal limits. Twenty-four hour urine revealed $693 \mathrm{mg}$ of protein, without any abnormal peaks identified on urine protein electrophoresis. Urine drug screen was negative.

During his fourth admission, he stated that each time he had these symptoms, and he was compelled to bathe almost continuously. Remarkably, the patient had made an association between his vomiting, compulsive bathing and renal impairment. Throughout admission, it was noted that he was almost invariably in the shower. Four days after discharge, he was readmitted for the fifth time with severe nausea, vomiting, lethargy, BUN of $121 \mathrm{mg} / \mathrm{dl}$ and creatinine of $9.09 \mathrm{mg} /$ dl. He only required intravenous hydration and in less than 24 hours, his serum creatinine had decreased to $2.23 \mathrm{mg} / \mathrm{dl}$. Compulsive bathing was again observed. By the third day, his creatinine had returned to normal. Human immunodeficiency virus testing was negative and gastric emptying study was normal. His presentation was consistent with cannabinoid hyperemesis syndrome. He was offered counselling for his marijuana addiction but stated he was able to quit on his own. Six months after discharge, he had not returned to hospital. 


\section{Discussion}

Since its first description by Allen et al [1], CHS has generated significant interest in the medical literature. In 2009, Sontineni et al proposed essential, major and supportive diagnostic criteria [2]. Simonetto et al expanded the diagnostic criteria but maintained the format proposed by Sontineni et al [3]. Current hypotheses for the pathophysiology suggest roles for $\mathrm{CB} 1$ and $\mathrm{CB} 2$ cannabis receptors in the central nervous and peripheral enteric systems, as well as presynaptic parasympathetic ganglia $[3,4]$.

Few reports have emphasized a significant association with acute renal impairment. Price et al [5] and Bramstedt et al [6] reported acute renal impairment that resolved completely with hydration. Electrolyte abnormalities include hyponatremia, hypochloremia, metabolic alkalosis and hypokalemia $[5,7]$. Our patient had significant elevation of blood urea nitrogen and creatinine, hyponatremia, hypochloremia, hypokalemia, hypophosphatemia, hypomagnesemia and recurrent mild rhabdomyolysis. The most consistent features at the time of presentation were hypochloremia, normal or low serum bicarbonate and a high anion gap. Except in the second hospitalization, hyponatremia was always present at the time of admission. Patients with severe vomiting typically develop hypochloremic hypokalemic metabolic alkalosis due to significant loss of hydrogen and chloride rich gastric fluid, with concomitant increase in serum bicarbonate to replace lost anions. Sodium and potassium ions present in significant amounts in gastric fluid are also lost through vomiting, leading to hyponatremia and hypokalemia [8]. The finding of a normal serum bicarbonate level in our patient was due to a mixed acid base disturbance in the presence of acute renal failure with uremia. We propose that the metabolic hallmark of this disorder is hypochloremic hyponatremic dehydration with mixed metabolic alkalosis and high anion gap metabolic acidosis when renal failure is present.

Urinary findings included proteinuria and microscopic hematuria on routine urinalysis during all his admissions. At discharge after the fifth admission, proteinuria had resolved. Although rare, renal effects of cannabis reported in the literature include post-transplantation membranous glomerulonephritis [9] and acute renal infarction [10].

As noted, a role has been suggested for peripheral eneteric nervous system $\mathrm{CB} 1$ and $\mathrm{CB} 2$ receptors, in the pathophysiology of persistent vomiting [3]. Endoscopic findings reported in this syndrome include esophagitis and gastric mucosal trauma from retching [7], as also seen in our case. Delayed gastric emptying has been reported during acute attacks, which resolves shortly after cessation of cannabis use. Our patient's gastric emptying study performed during his fifth admission was however normal, with gastric retention of $95 \%$ at 30 minutes and $2 \%$ at 3 hours. In the series by
Simonetto et al, gastric emptying was either normal or rapid in majority of patients [3].

\section{Conclusions}

Our case report highlights a serious and potentially lifethreatening complication of a recently recognized disorder. It is unclear if our patient's findings are solely due to severe volume depletion, whether other mechanisms play an equal or more important role or if the course of renal involvement is benign in the long term. Other reports will be required to further elucidate the nature of renal involvement.

\section{References}

1. Allen JH, de Moore GM, Heddle R, Twartz JC. Cannabinoid hyperemesis: cyclical hyperemesis in association with chronic cannabis abuse. Gut. 2004;53(11):15661570 .

2. Sontineni SP, Chaudhary S, Sontineni V, Lanspa SJ. Cannabinoid hyperemesis syndrome: clinical diagnosis of an underrecognised manifestation of chronic cannabis abuse. World J Gastroenterol. 2009;15(10):1264-1266.

3. Simonetto DA, Oxentenko AS, Herman ML, Szostek JH. Cannabinoid hyperemesis: a case series of 98 patients. Mayo Clin Proc. 2012;87(2):114-119.

4. Patterson DA, Smith E, Monahan M, Medvecz A, Hagerty B, Krijger L, Chauhan A, et al. Cannabinoid hyperemesis and compulsive bathing: a case series and paradoxical pathophysiological explanation. J Am Board Fam Med. 2010;23(6):790-793.

5. Price SL, Fisher C, Kumar R, Hilgerson A. Cannabinoid hyperemesis syndrome as the underlying cause of intractable nausea and vomiting. J Am Osteopath Assoc. 2011;111(3):166-169.

6. Bramstedt J, Dissmann R. [Cannabinoid hyperemesis syndrome inducing acute prerenal failure and electrolyte disturbance]. Dtsch Med Wochenschr. 2011;136(3435):1720-1722.

7. Sullivan S. Cannabinoid hyperemesis. Can J Gastroenterol. 2010;24(5):284-285.

8. Gennari FJ, Weise WJ. Acid-base disturbances in gastrointestinal disease. Clin J Am Soc Nephrol. 2008;3(6):1861-1868.

9. Bohatyrewicz M, Urasinska E, Rozanski J, Ciechanowski K. Membranous glomerulonephritis may be associated with heavy marijuana abuse. Transplant Proc. 2007;39(10):3054-3056.

10. Lambrecht GL, Malbrain ML, Coremans P, Verbist L, Verhaegen $\mathrm{H}$. Acute renal infarction and heavy marijuana smoking. Nephron. 1995;70(4):494-496. 